\title{
Alterations in the memory code for temporal events induced by differential outcome expectancies in pigeons
}

\author{
MARY JO DUCHARME and ANGELO SANTI \\ Wilfrid Laurier University, Waterloo, Ontario, Canada
}

\begin{abstract}
The effect of differential outcome expectancies on memory for temporal and nontemporal information was examined. Pigeons were trained to match short (2-sec) and long (8-sec) sample durations to red and green comparison stimuli, and vertical and horizontal lines to vertical and horizontal comparison stimuli. In Experiment 1, one differential outcome (DO) group received food for correct choices on short-sample trials, whereas another received food for correct choices on long-sample trials. On line-orientation trials, half of each DO group received food for correct responses following vertical samples, whereas the other half received food for correct responses following horizontal samples. Overall retention was greater in the DO groups than in a nondifferential (NDO) group that received either food or no food for correct responses on a random half of all trials. Furthermore, although the NDO group displayed a choose-short bias for temporal samples, both DO groups displayed equivalent biases to select the comparison stimulus associated with food. In Experiment 2, differential outcome expectancies were extinguished off-baseline. Subsequently, in the first nondifferential outcome test session, the DO groups performed less accurately than the NDO group. These findings indicate that temporal samples are not retrospectively and analogically coded when they are differentially associated with food and no food. Instead, they are remembered in terms of the corresponding outcome expectancies.
\end{abstract}

The delayed matching-to-sample (DMTS) procedure has been used extensively to study working memory processes in animals. After a delay interval, subjects are required to match one of two comparison stimuli to a previously presented sample stimulus in order to receive the reinforcer. As the delay between the sample stimulus and the comparison stimuli is increased, matching accuracy declines. However, when different types of reinforcers are associated with correct matching responses to each sample stimulus, retention over the delay interval is better than when the same type of reinforcer is used after each sample stimulus. This differential outcome effect (DOE) has also been manifested as increased rates of acquisition, higher levels of overall accuracy, and a bias to choose the comparison stimulus associated with the more favorable outcome (Brodigan \& Peterson, 1976; DeLong \& Wasserman, 1981; Edwards, Jagielo, Zentall, \& Hogan, 1982; Honig, Matheson, \& Dodd, 1984; Nevin \& Grosch, 1990; Peterson, 1984; Peterson \& Trapold, 1980; Peterson, Wheeler, \& Armstrong, 1978; Peterson, Wheeler, \& Trapold, 1980; Santi, 1989; Santi \& Roberts,

This research was supported by Grant $\mathrm{A} 6378$ from the Natural Sciences and Engineering Research Council of Canada to A.S. This article is based on a master's thesis submitted by the first author to Wilfrid Laurier University. Reprints may be obtained from A. Santi, Department of Psychology, Wilfrid Laurier University, Waterloo, ON, Canada N2L 3C5. 1985a, 1985b; Urcuioli, 1990a, 1990b, 1991; Williams, Butler, \& Overmier, 1990).

The superior performance associated with the DOE has been commonly explained by the idea that, through experience with the samples and their correlated outcomes, subjects develop sample-specific expectancies of those outcomes. In turn, the outcome expectancies cue correct choice behavior (Peterson, 1984; Trapold, 1970; Urcuioli, 1990a, 1991). The degree of control maintained by the sample stimuli in a differential outcome (DO) situation has been debated. However, Urcuioli (1990b) presented evidence suggesting that differential outcome expectancies of food and no food overshadow the control by the sample stimuli over choice, whereas expectancies of different probabilities of reinforcement do not. Therefore, depending on the salience of the outcomes, the sample stimuli play a role of variable importance.

Studies involving temporal samples provide a useful perspective on the degree of control maintained by the sample stimuli in a DO situation. A phenomenon known as the choose-short bias arises in DMTS when variablelength delay intervals are presented following temporal samples. As the delay interval increases, pigeons show an increasing tendency to peck the comparison stimulus corresponding to the short-duration sample (Spetch, 1987; Spetch \& Rusak, 1989; Spetch \& Wilkie, 1982, 1983). The nature of the memory code for temporal samples that gives rise to the choose-short effect has been debated (Kraemer, Mazmanian, \& Roberts, 1985). However, most 
research suggests that when no other salient cues are available, subjects maintain a memory of the actual sample duration over the delay interval (Spetch \& Sinha, 1989; Wilkie \& Willson, 1990).

A study by Santi, Ducharme, and Bridson (1992) that examined DO expectancies and memory for temporal and nontemporal stimuli found evidence consistent with the conclusion drawn by Urcuioli (1990b). Different probabilities of reward were correlated with short (2-sec) and long (8-sec) houselight samples, as well as with vertical and horizontal line samples. When the sample stimuli were nontemporal, subjects displayed a comparison response bias to the more favorable outcome (i.e., to the comparison associated with the higher probability of reinforcement). This bias did not increase as the delay interval was increased. When the sample stimuli were temporal, differential reinforcement probabilities enlarged the chooseshort effect relative to a nondifferential group when the high probability was associated with short-duration samples, but eliminated it when the high probability was associated with long-duration samples. The enhanced choose-short effect exhibited by the subjects receiving the high probability of reinforcement after the short sample stimulus suggested that the bias to choose short was working in conjunction with the bias to choose favorably (short) and the DO expectancy to choose correctly (short). The diminished effect shown by subjects receiving the high probability of reinforcement after the long sample stimulus indicated that the bias to choose favorably (long) and the DO expectancy to choose correctly (long) counteracted the bias to choose short. Santi et al. (1992) concluded, then, that when different probabilities of reinforcement are associated with temporal samples, the working memory code is neither simply an outcome expectancy nor simply a retrospective and analogical code of event duration.

Although expectancies based on different probabilities of reward do not appear to dominate the control established by temporal or nontemporal sample stimuli, Urcuioli's (1990b) results indicate that the expectation of food versus no food might well do so. Consequently, in the present study, memory for event durations in pigeons was assessed with food versus no food as outcomes for correct responses. With these outcomes, control by temporal samples may be overridden by a food versus nofood expectancy code, resulting in retention gradients for temporal samples that do not reflect the operation of a subjective shortening mechanism. Instead, the retention gradients should reflect the operation of outcome expectancies associated with the temporal samples.

\section{EXPERIMENT 1}

Pigeons were trained on DMTS with short- and longduration samples mapped onto red and green comparison stimuli, and vertical and horizontal lines mapped onto vertical and horizontal comparison stimuli. Three groups of birds were employed, two of which received differential outcomes. The DO-SF (differential outcome-short food) group received food for correctly responding on short-duration temporal sample trials and no food (the hopper light with no food access) for correctly responding on long-duration temporal sample trials. The DO-LF (differential outcome-long food) group received food for correctly responding on long-duration trials, and no food for correctly responding on short-duration trials. On nontemporal trials, half of each DO group received food reinforcement for correct responses on vertical trials, while the other half received it on horizontal trials. A nondifferential outcome (NDO) group received food on a random half of all correctly completed line- and durationsample trials, and no food on the remaining correctly completed trials.

Considering the previous finding of Urcuioli (1990b) with respect to food and no-food outcome expectancies, it was predicted that a DOE would develop in the two DO groups and that the food versus no-food expectancies would mask the control of both the temporal samples and the line-tilt samples. Three findings would be consistent with this prediction. First, the overall performance of the DO groups would be superior to that of the NDO group. Second, although the NDO group should display a typical choose-short bias on the temporal dimension, performance in the DO groups on the temporal dimension should predominantly reflect a bias to peck the comparison stimulus associated with food. This choose-favorable-outcome bias should produce higher accuracy on short-sample trials in the DO-SF group and higher accuracy on long-sample trials in the DO-LF group. Third, because expectancies should guide choice behavior more than should the sample stimuli themselves in the DO groups, their retention functions should be similar for both temporal and nontemporal samples, and an equivalent bias to select the comparison stimulus associated with the favorable outcome should be observed.

\section{Method}

\section{Subjects}

A total of 24 DMTS-experienced White Carneaux pigeons, maintained at approximately $80 \%$ of their ad-lib weights and housed individually with grit and water continuously available, served as subjects. They were exposed to a 12:12-h light:dark cycle. The experimental sessions took place between $1200 \mathrm{~h}$ and $1700 \mathrm{~h}$ of the subjects' light cycle, 5 to 7 days a week.

\footnotetext{
Apparatus

Four Coulbourn modular operant chambers (Model E10-10), contained in isolation chambers (Model E10-20) that were equipped with ventilation fans and baffled air intakes and exhaust systems, were used. Each chamber contained three horizontally aligned, clear plastic keys behind which projectors could display stimuli (red or green, white vertical or horizontal line on a black background, or a black dot on a white background) onto a frosted rear projection screen (Coulbourn Model E21-18). A houselight was located $6.5 \mathrm{~cm}$ above the center key; it was installed so that the light was directed upward to reflect from the top of the cage (Coulbourn Model E11-01 with bulb SL1819X). Directly below the center key was a $5.7 \times 5 \mathrm{~cm}$ opening that provided access to a hopper filled with mixed grain (Coulbourn Model E14-10). All experimental events were controlled by a microcomputer located in an adjacent room.
} 


\section{Procedure}

Pretraining. Because of the different experimental histories of the 24 birds, a lengthy phase (40 sessions) of DMTS pretraining was undertaken. Line-tilt (vertical and horizontal lines) and temporal (2-sec and 8-sec houselight duration) samples were used, and their occurrence was randomized over trials. Line-tilt sample stimuli were mapped onto line-tilt comparisons, and temporal sample stimuli were mapped onto red and green comparison stimuli. Position of the comparison stimuli (left or right) was counterbalanced over trials. On nontemporal-sample trials, a warning signal (a small black dot on a white background) was presented on the center key. A peck to it resulted in the presentation of either a vertical- or a horizontalline sample stimulus on the center key. The line-tilt sample was presented for $4 \mathrm{sec}$ (the geometric mean of the houselight sample durations) and was followed immediately by presentation of vertical and horizontal lines as comparison stimuli. On temporal-sample trials, the trial began immediately with illumination of the houselight sample for $2 \mathrm{sec}$ or $8 \mathrm{sec}$. The termination of the temporal sample was followed by the presentation of red and green lights on the side keys. Of the 24 birds, 12 were required to peck red after a short-duration sample stimulus and green after a long-duration sample stimulus, and 12 were required to peck green after short and red after long. During this phase, all correct responses were rewarded with 3-sec access to mixed grain from an illuminated hopper.

Intertrial intervals were a constant $15 \mathrm{sec}$ in length. Each session consisted of 80 trials, $\mathbf{4 0}$ with temporal samples and $\mathbf{4 0}$ with line-orientation samples. A correction procedure was in effect throughout the duration of pretraining so that selection of the incorrect comparison stimulus produced a 3-sec blackout followed immediately by re-presentation of the same sample stimulus. A correct response on a correction trial produced 3-sec access to mixed grain, although only the choice response on the initial (noncorrection) trial was used to calculate matching accuracy. Although the subjects were not assigned to the different reinforcement outcome conditions until after the pretraining phase, an analysis of the performance levels at the end of pretraining was undertaken as a function of the groups to which the subjects were eventually assigned. The mean performance levels on the temporal dimension over the last three days of pretraining were $94.3 \%, 95.1 \%$, and $93.5 \%$ correct for the DO-SF, DO-LF, and NDO groups, respectively. On the nontemporal dimension, the corresponding mean performance levels over the last three days were $84.2 \%, 77.9 \%$, and $81.0 \%$ correct, respectively. An analysis of variance (ANOVA) revealed no significant differences between groups $[F(2,21)<1]$ and no group $x$ dimension interaction $[F(2,21)<1]$. However, accuracy averaged across groups was higher on the temporal than on the nontemporal dimension $[F(1,21)=14.14, p<.01]$.

Training. All 24 birds were then trained on a DMTS procedure with parameters identical to the pretraining ones except for the reinforcement condition following correct responses. All 8 birds in the DO-SF group received 3-sec access to food for correct responses on short-sample trials and no food (3-sec presentation of the hopper light with no access to mixed grain) for correct responses on long-sample trials. The 8 birds in the DO-LF group received food for correct responses on long-sample trials and no food for correct responses on short-sample trials. On nontemporal-sample trials, half of the birds in each DO group received food for correct responses on vertical-sample trials and no food for correct responses on horizontal-sample trials. The remaining birds received food on horizontal-sample trials and no food on vertical-sample trials. The 8 NDO (nondifferential outcome) birds received food and no food randomly following correct responses on both temporal- and nontemporal-sample trials. The correction procedure remained in effect during training primarily to maintain reasonable levels of accuracy on the no-food outcome trials in the DO groups. Correct responses on correction trials produced the outcomes appropriate to the group conditions described above. Intertrial intervals remained at $15 \mathrm{sec}$, and each session consisted of 80 trials. Training continued for 25 sessions.

Delay testing. Delay-interval testing was conducted for 20 sessions following training. Each session consisted of 96 trials. A 0-sec delay occurred on $75 \%$ of the trials, and delays of 5,10 , and $15 \mathrm{sec}$ occurred equally on the remaining $25 \%$ of the trials. All other parameters were identical to the training phase.

\section{Results}

The mean performance levels for each group over the last three days of training on the temporal dimension were $88.0 \%, 85.8 \%$, and $95.7 \%$ correct for the DO-SF, DO-LF, and NDO groups, respectively. On the nontemporal dimension, the corresponding means were $84.6 \%, 73.8 \%$, and $87.1 \%$ correct, respectively. An ANOVA revealed a main effect of group $[F(2,21)=3.50, p<.05]$. Subsequent Newman-Keuls tests revealed that the NDO group performed more accurately than the DO groups. In addition, although overall accuracy was higher on the temporal than on the nontemporal dimension $[F(1,21)=$ $16.75, p<.001]$, there was no group $\times$ dimension interaction $[F(2,21)=1.61]$.

To establish whether there were significant overall effects associated with differential outcomes during delay testing, the data were collapsed across sample type (short/long and vertical/horizontal) and are shown in Figure 1. The upper graph presents the data for temporalsample trials, and the lower graph presents the data for nontemporal-sample trials. On both types of trials, the two DO groups performed more accurately than the NDO group at delays greater than $0 \mathrm{sec}$. This demonstrates the usual beneficial effect of differential outcomes on memory performance. An ANOVA with group (DO-SF, DO-LF, and NDO), dimension (temporal and nontemporal), and delay $(0,5,10$, and $15 \mathrm{sec})$ as factors revealed main effects of group $[F(2,21)=14.40, p<.0001]$ and delay $[F(3,63)=165.87, p<.0001]$, as well as a delay $\times$ group interaction $[F(6,63)=26.70, p<.0001]$. Newman-Keuls tests at the .05 confidence level confirmed that at the 5-, 10-, and 15-sec delay intervals, the two DO groups outperformed the NDO group. Although overall accuracy was slightly higher on the temporal $(\mathbf{7 6 . 2 \% )}$ than on the nontemporal $(73.1 \%)$ dimension, the difference was not statistically significant $[F(1,21)=3.63, p=.07]$.

To assess the effects of differential outcome expectancies on memory for temporal and nontemporal events in more detail, the data for the two DO groups were analyzed with outcome (food/no food) as a factor. The top and bottom halves of Figure 2 display the data for temporal and nontemporal samples, respectively. Across the various delays, both DO groups displayed a bias to select the comparison stimulus associated with the more favorable (food) outcome regardless of whether the samples were temporal or nontemporal. On temporal-sample trials, this choose-favorable-outcome bias produced higher accuracy on short-sample trials in the DO-SF group and higher accuracy on long-sample trials in the DO-LF group. An ANOVA with group (DO-SF and DO-LF), 
TEMPORAL SAMPLES
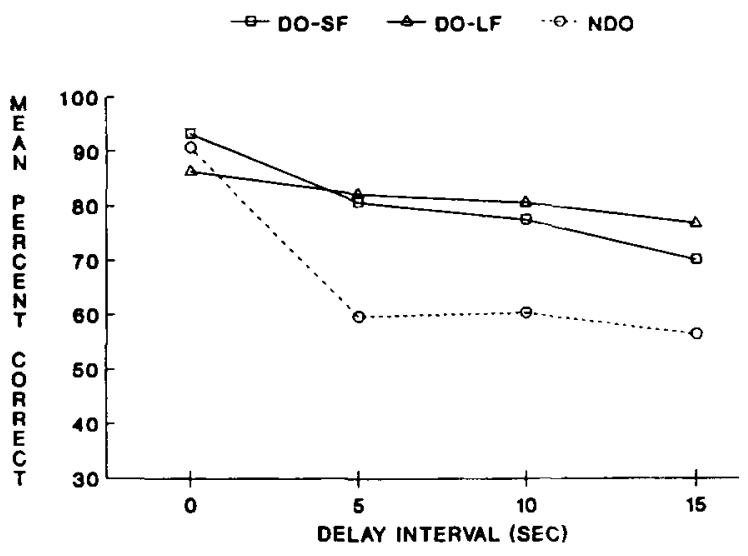

NONTEMPORAL SAMPLES

$\rightarrow$ DO-SF $\rightarrow$ DO-LF $\quad$ O. NDO

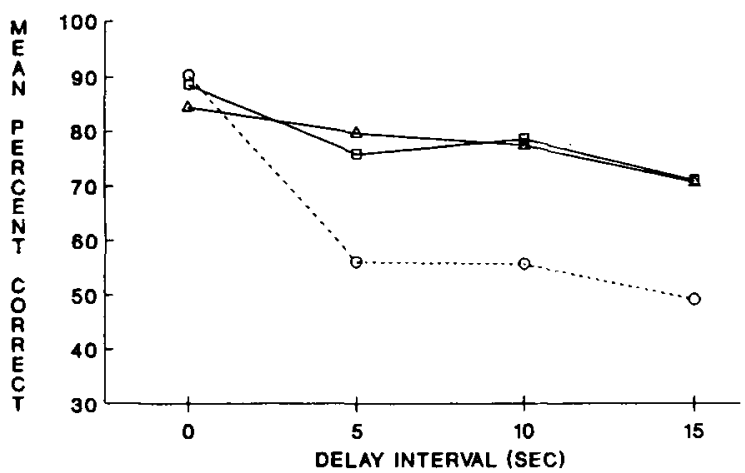

Figure 1. Mean percentage of correct responses by delay for temporal and nontemporal samples for each group (DO-SF), DO-LF, and NDO) in Experiment 1.

dimension (temporal and nontemporal), outcome (food and no food), and delay $(0,5,10$, and $15 \mathrm{sec})$ as factors indicated that accuracy was significantly greater when the trial outcome was food than when it was no food $[F(1,14)=87.14, p<.0001]$. Matching accuracy decreased significantly as the delay interval increased $[F(3,42)=57.29, p<.0001]$. There was no significant overall difference between the DO-SF and DO-LF groups $[F(1,14)<1]$, and the interaction of group $\times$ dimension $\times$ outcome $\times$ delay was not significant $[F(3,42)=$ 1.68]. However, there was a group $\times$ outcome $\times$ delay interaction $[F(3,42)=6.15, p<.01]$, which was primarily due to a significant interaction of group $\times$ delay when the outcome was no food $[F(3,42)=6.70, p<$ $.001]$. This in turn was due to the DO-SF group tending to perform more accurately than the DO-LF group at the 0 -sec delay interval when the outcome was no food $[F(1,14)=4.12, p=.06]$.

The data for the DO groups on the temporal-sample trials are redisplayed in Figure 3 along with those for the NDO group. Although the NDO group displayed a typical choose-short bias, performance in the DO groups predominantly reflected a bias to peck the comparison stimulus associated with food. This choose-favorableoutcome bias resulted in higher accuracy on short-sample trials in the DO-SF group and higher accuracy on longsample trials in the DO-LF group across all delay intervals, including $0 \mathrm{sec}$. An ANOVA with group (DO-SF, DO-LF, and NDO), duration (short and long), and delay $(0,5,10$, and $15 \mathrm{sec})$ as factors was performed and revealed a three-way interaction of group $x$ duration $x$ delay $[F(6,63)=9.34, p<.0001]$. For the NDO group, accuracy on the short-sample trials was significantly higher than it was on long-sample trials at the 15-sec delay $[F(1,21)=27.38, p<.0001]$, the 10 -sec delay $[F(1,21)=$ $18.63, p<.001]$, and the 5-sec delay $[F(1,21)=9.43$, $p<.01]$ intervals, but not at the 0 -sec delay interval $[F(1,21)<1]$. In addition, accuracy declined as the delay interval was increased on both long-sample
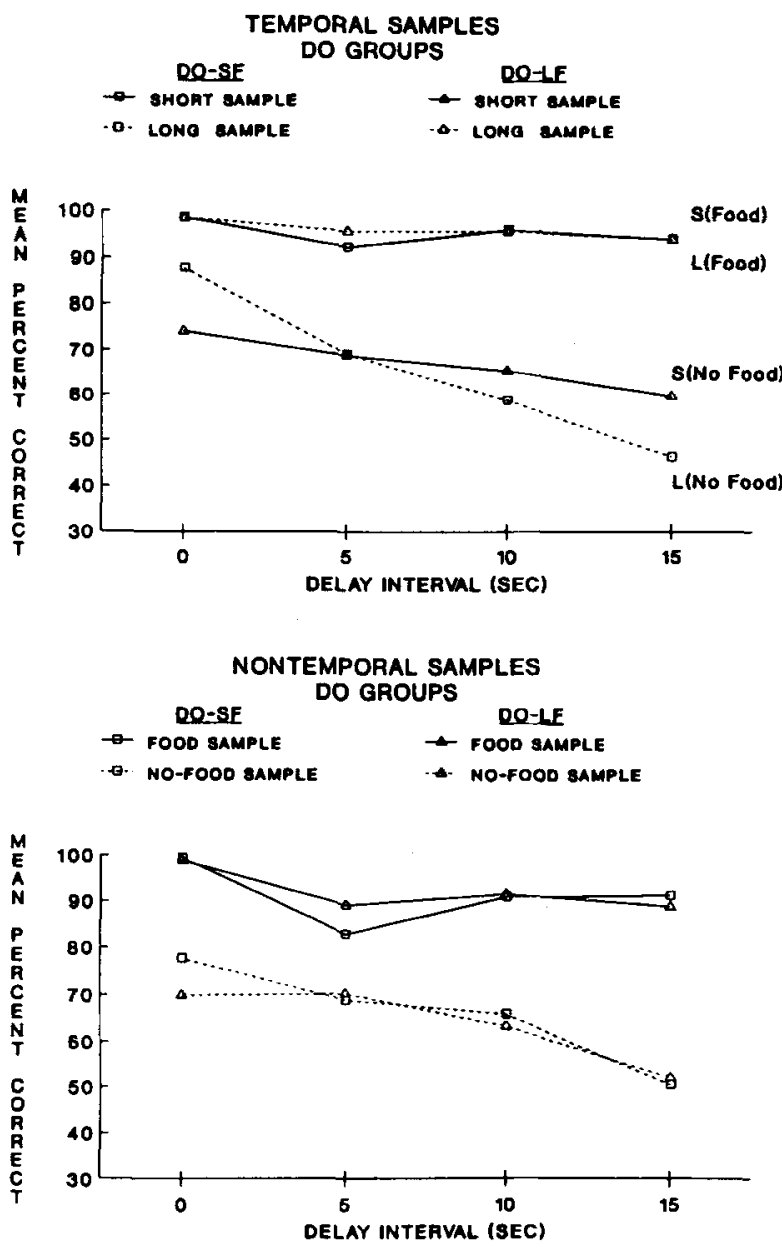

Figure 2. Mean percentage of correct responses for temporal and nontemporal samples as a function of differential outcome group (DO-SF and DO-LF) and sample type during delay interval testing in Experiment 1. The outcome associated with short or long samples is noted in parentheses in the top panel. For nontemporal samples, the data are reported for the visual sample associated with the food outcome (food sample) and for the visual sample associated with the no-food outcome (no-food sample). 

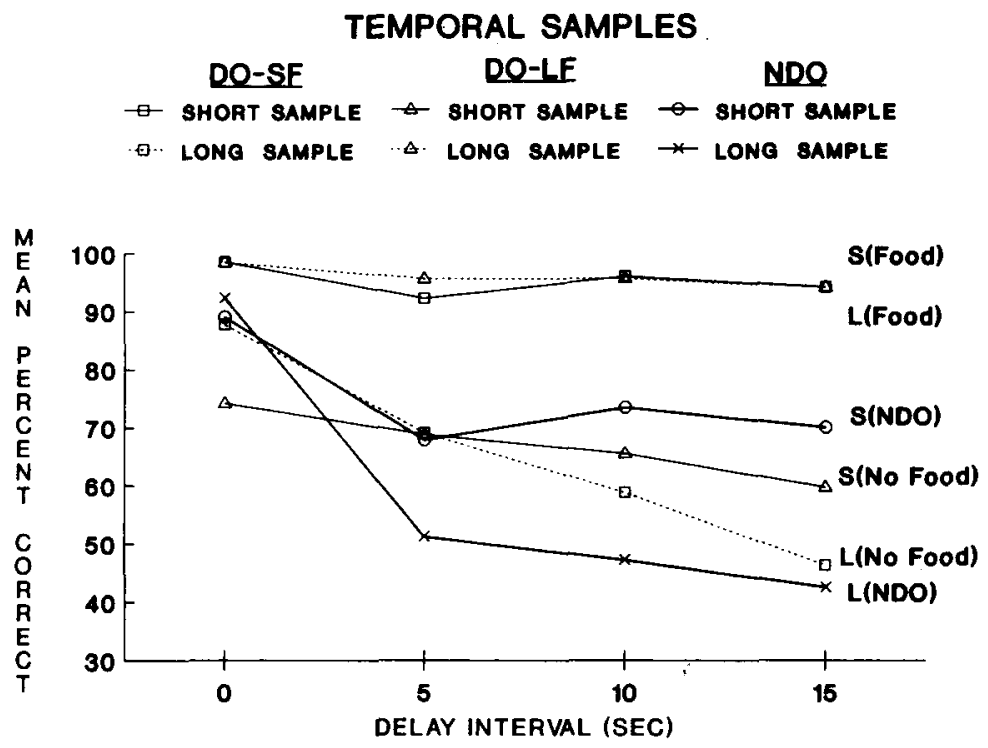

Figure 3. Mean percentage of correct responding for temporal samples as a function of group (DO-SF, DO-LF, and NDO) sample duration (short of long), and delay interval $(0,5,10$, and $15 \mathrm{sec})$ in Experiment 1. The outcome associated with short or long samples for the groups is noted in parentheses.

$[F(3,63)=80.50, p<.0001]$ and short-sample $[F(3,63)=14.04, p<.0001]$ trials. For the DO-SF group, a bias to peck the comparison stimulus associated with the short-duration sample and with the favorable outcome (food) was significant at all delay intervals, including $0 \mathrm{sec}[F \mathrm{~s}(1,21)=9.79,18.38,37.39,82.77$, respectively]. For the DO-SF group, accuracy declined as the delay interval increased only on the long-sample (no-food) trials $[F(3,63)=47.10, p<.0001]$. For the DO-LF group, a significant bias to peck the comparison stimulus associated with the long-duration sample and with the favorable outcome (food) occurred at the 0-, 5-, 10-, and 15-sec delays $\left[F_{\mathrm{S}}(1,21)=42.78,24.58,24.80\right.$, 48.96, respectively]. In addition, accuracy declined as the delay interval increased only on the short-sample (nofood) trials $[F(3,63)=5.63, p<.01]$. Thus, accuracy declined in both DO groups, with increasing delay only on trials for which the outcome was no food.

In summary, these analyses indicate that in addition to the usual beneficial effects of differential outcomes on retention, the DO groups generally demonstrated an equivalent choose-favorable-outcome bias on both the temporal and nontemporal dimensions, whereas the NDO group displayed the typical choose-short bias following temporal samples.

\section{Discussion}

The findings of Experiment 1 are consistent with the hypothesis that outcome expectancies are controlling choice behavior and are replacing the control previously established by temporal and nontemporal samples. The overall superior performance of the DO groups relative to the NDO group during delay testing suggests that dif- ferential outcome expectancies facilitated correct choices. As noted in the procedure section, however, the performance of the NDO group was significantly more accurate than that of the DO groups at the end of the 25 differentialoutcome training sessions prior to delay testing. This difference reflected a drop in the performance of the DO groups during differential-outcome training relative to their performance during the preceding nondifferentialoutcome pretraining phase. The drop may have reflected a shift in the control of choice behavior from sample stimuli to outcome expectancies that was not quite completed in the $\mathbf{2 5}$ training sessions. With continued exposure to the 0 -sec-delay condition during delay testing, however, the performance of the DO groups improved such that there was no longer a significant difference between their zero-delay performances and those of the NDO group.

At delay intervals greater than $0 \mathrm{sec}$, the DO-SF and DO-LF groups performed similarly to each other on both food and no-food outcome trials regardless of whether these outcomes were associated with temporal or nontemporal samples. This suggests that sample-stimulus information was being replaced by the birds' outcome expectancies and that the difference in accuracy on food and no-food outcome trials was due to a choose-favorableoutcome bias. Thus, although the NDO group displayed a typical choose-short effect on temporal sample trials, the DO-SF and DO-LF groups both displayed a choosefavorable-outcome bias. Note that in a design similar to the present study, Santi et al. (1992) found that differential probabilities of reinforcement did not result in the two DO groups performing similarly to each other. In that study, associating a high probability of reinforcement with the short sample enhanced the choose-short effect. Associating it with the long sample eliminated the effect but 
did not produce a choose-long effect. In view of this, the present data suggest that the food and no-food outcomes used here were more salient than the different probabilities of reward used by Santi et al. (1992).

\section{EXPERIMENT 2}

In Experiment 2, differential outcome expectancies were removed by pairing nondifferential outcomes with both temporal and nontemporal sample stimuli in the DO groups. This was accomplished out of the matching context, or "off-baseline," to avoid possible disruptions in performance that could occur if differential outcomes were immediately removed from the discrimination. During offbaseline training, all of the subjects were presented with the sample stimuli (vertical and horizontal lines, and 2 and $8 \mathrm{sec}$ of houselight illumination), which were then immediately followed by either food or no food at random. No choice stimuli were presented during the 12 sessions of off-baseline training. Subsequently, all of the birds were returned to zero-delay matching-to-sample (MTS) with nondifferential outcomes for correct responding in order to assess the amount of control exercised by the sample stimuli themselves. Although all groups received nondifferential outcomes in Experiment 2, the groups will still be referred to as DO and NDO to represent their relevant history from Experiment 1.

The data from Experiment 1 suggested that sample stimulus control was replaced by differential outcome expectancies. If this interpretation is correct, then removal of the expectancies should produce less accurate responding in the DO group than in the NDO group when zerodelay MTS is reintroduced. Following off-baseline training, the performance of the NDO group should remain accurate because the same controlling cues are active, whereas the accuracy of the DO groups should decline. On the other hand, if our interpretation of the results of Experiment 1 is incorrect, and differential outcome expectancies did not alter control by the sample stimuli, then removal of the DO expectancies in Experiment 2 should produce equivalent performances by the DO and NDO groups when zero-delay MTS is reintroduced.

\section{Method \\ Subjects and Apparatus \\ The subjects were the same as those in Experiment 1, except for 1 subject from the original DO-LF group that was dropped because of illness. The apparatus was the same as that in Experiment 1.}

\section{Procedure}

Return to baseline. This phase was identical to the training phase of Experiment 1. It began 21 days after Experiment 1 concluded and was carried out for 7 days. The mean performance level on the temporal dimension over the last 3 days of this phase was $92.0 \%$ correct for the combined DO groups and $92.9 \%$ correct for the NDO group. On the nontemporal dimension, the mean performance level over the last 3 days was $87.7 \%$ correct for the combined DO groups and $91.5 \%$ correct for the NDO group. An ANOVA revealed no significant difference between groups $[F(1,21)=1]$ and no group $x$ dimension interaction $[F(1,21)<1]$. However, accuracy aver- aged across groups tended to be higher on the temporal than on the nontemporal dimension $[F(1,21)=4.00, p=.06]$.

Off-baseline training. In these sessions, all of the subjects were exposed to sample stimuli (vertical and horizontal lines for $4 \mathrm{sec}$ and houselight illumination for 2 and $8 \mathrm{sec}$ ) that were immediately followed by either food (3-sec access to mixed grain in an illuminated hopper) or no food (illuminated hopper light only) each on a random half of all trials. This phase continued for 12 sessions of 80 trials each. Urcuioli (1990b) reported that 3-12 sessions of off-baseline training were required to ensure that the birds were responding nondifferentially to the sample stimuli. No data were recorded for these sessions.

Nondifferential outcome test phase. In this phase, the subjects were returned to the 0 -sec-delay matching situation with vertical and horizontal lines (presented for $4 \mathrm{sec}$ and preceded by a warning light as in Experiment 1) and 2 and $8 \mathrm{sec}$ of houselight illumination presented as samples. Duration samples continued to be mapped onto red and green comparison stimuli, and line-tilt samples were mapped onto line-tilt comparison stimuli. Food and no-food outcomes were presented nondifferentially such that each outcome followed a correct response equally often. Also, a correction procedure identical to that used in Experiment 1 remained in effect. This phase continued for 10 sessions of 80 trials each.

Delay testing. This phase was identical to the last except that delay intervals of 5,10 , and $15 \mathrm{sec}$ were randomly presented on 24 of the 96 trials. On 72 of the 96 trials, a 0 -sec delay interval was used. Nondifferential outcomes identical to the previous phase were given following correct responses. Delay testing continued for 20 sessions.

\section{Results}

Figure 4 displays the data for the DO and NDO groups on both temporal (top panel) and nontemporal (bottom panel) samples averaged over the last 3 days of return to baseline (B), the first 3 days of nondifferential testing, and the last nondifferential test session (L). Because the DO-SF and DO-LF groups were treated equally from the point at which differential expectancy cues were removed, the data of these two groups were combined into one "DO' group $(n=15)$ for comparison with the data in the NDO group $(n=8)$. As can be seen in both the top and bottom panels of Figure 4, matching accuracy of the DO group on the first session of nondifferential testing was lower relative to that of the NDO group and relative to its previous baseline performance with differential outcomes. An ANOVA with dimensions (temporal and nontemporal), groups (DO and NDO), and the first three nondifferential test sessions as factors indicated a significant group $\times$ session interaction $[F(2,42)=4.47, p<$ .05]. An analysis of simple main effects revealed that the NDO group matched more accurately than the DO group on the first nondifferential test session $[F(1,21)=4.39$, $p<.05]$ but not on the second $[F(1,21)<1]$ or the third session $[F(1,21)<1]$. An analysis of each of the remaining nondifferential test sessions (4-10) revealed that the groups did not significantly differ $[F(1,21)<1]$. In addition, the analysis indicated that matching was more accurate on temporal-sample trials than on nontemporalsample trials $[F(1,21)=7.90, p<.05]$.

To examine the changes that occurred between the last three baseline (B) sessions with differential outcomes and the first nondifferential test session, an additional analy- 
TEMPORAL SAMPLES

- $\odot$ - NDO GROUP $\rightarrow$ DO GAOUP

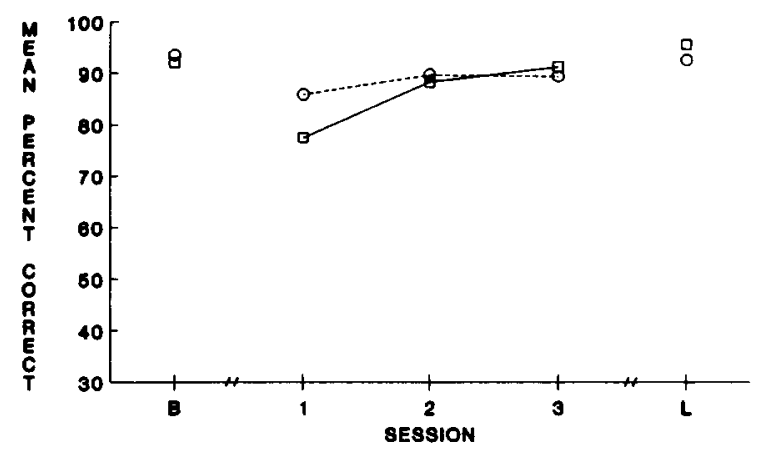

NONTEMPORAL SAMPLES

-Q- NDO GROUP $\longrightarrow$ DO GROUP

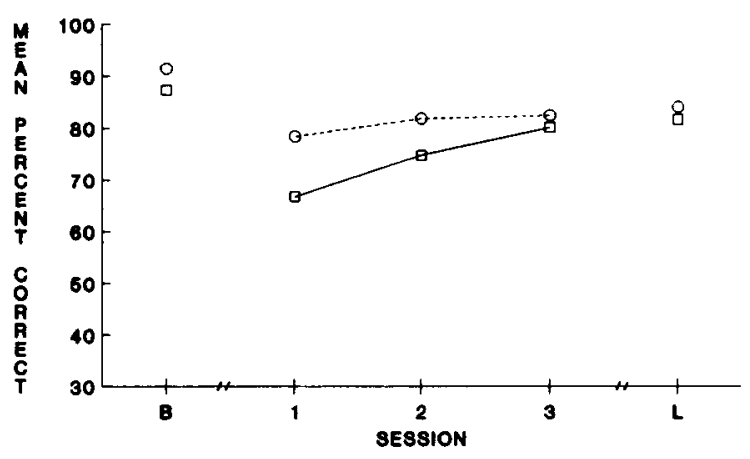

Figure 4. Nondifferential test performance for each group on temporal samples (top) and nontemporal samples (bottom) for the first three and last (L) 0-sec-delay test session in Experiment 2. Corresponding performance averaged over the last three sessions of the "return to baseline" (B) phase of Experiment 2 are also shown.

sis was performed. Groups (DO and NDO), session types (the last 3 days of baseline and the first nondifferential test session), and dimensions (temporal and nontemporal) were used as factors. The overall performance of the NDO group was significantly greater than that of the DO group $[F(1,21)=4.36, p<.05]$, and accuracy on the first nondifferential test session was lower than that on the last three sessions of baseline $[F(1,21)=34.70, p<.001]$. Finally, accuracy on temporal-sample trials was more accurate than that on nontemporal-sample trials $[F(1,21)=$ $6.18, p<.025]$. No other effects were significant.

Figure 5 shows the retention functions for the DO and NDO groups averaged over the 20 sessions of delay testing that followed the nondifferential test sessions. Similar choose-short biases on the temporal dimension for the DO and NDO groups can be seen in the upper panel of Figure 5, while the lower panel demonstrates the similarity of the groups on the nontemporal dimension. An ANOVA performed on these data included dimensions (temporal and nontemporal), groups (DO and NDO), and delay intervals $(0,5,10$, and $15 \mathrm{sec})$ as factors. Accuracy decreased significantly with increasing delay for both groups $[F(3,63)=236.87, p<.001]$. Also, a significant dimension $\times$ group interaction occurred $[F(1,21)=$ $5.64, p<.05]$. An analysis of simple main effects revealed that the DO group performed more accurately on temporal- than on nontemporal-sample trials $[F(1,21)=$ $34.54]$ and that the NDO group performed equivalently on both types of trials $[F(1,21)<2]$. However, there were no significant differences in accuracy between the DO and NDO groups on temporal-sample $[F(1,21)<3]$ or nontemporal-sample $[F(1,21)<2]$ trials.

To investigate the occurrence of a choose-short effect, a separate analysis was performed on the temporal-sample data with sample durations (short and long), delay intervals $(0,5,10$, and $15 \mathrm{sec})$, and groups (DO and NDO) as factors. There was no significant difference due to groups $[F(1,21)<3]$ or any significant interaction involving groups. However, there was a sample duration $x$ delay interval interaction $[F(3,63)=4.68, p<.01]$.
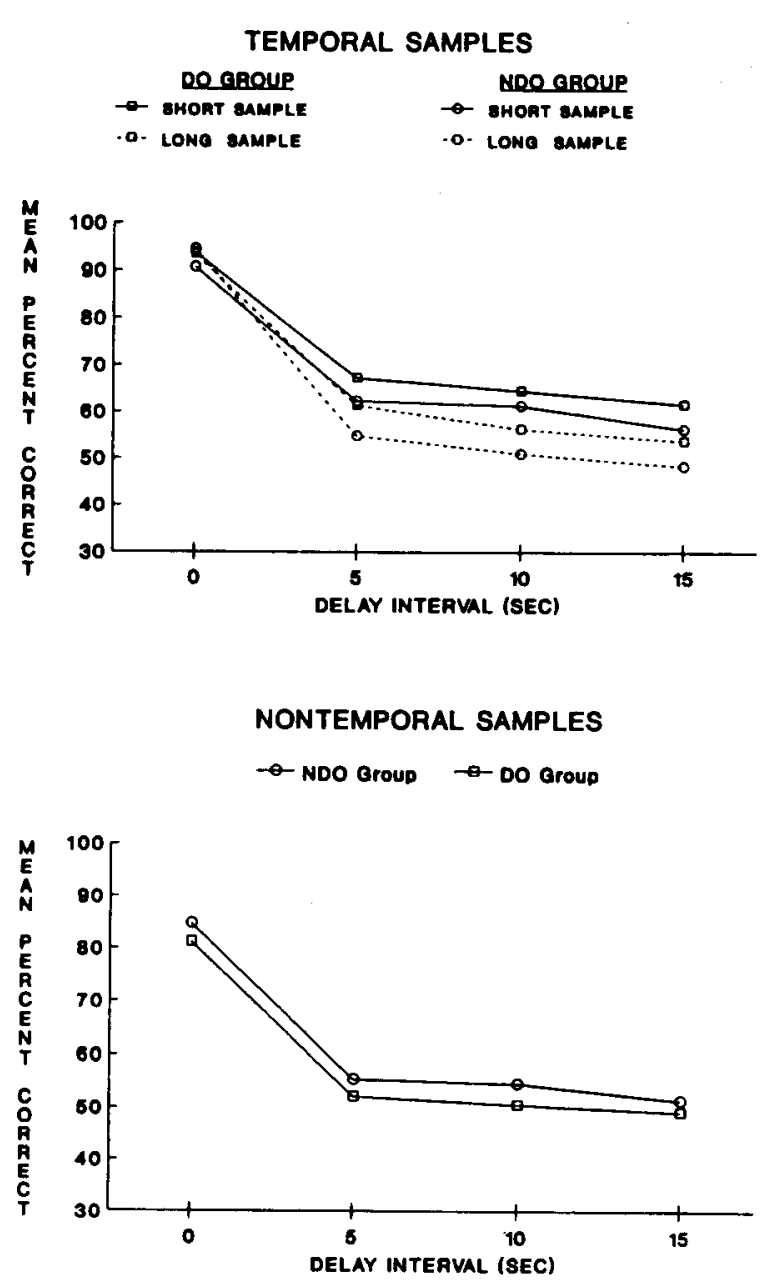

Figure 5. Mean percentage of correct responding for temporal samples (top) and nontemporal samples (bottom) as a function of previous differential outcome condition (DO and NDO) during delay testing with nondifferential reinforcement in Experiment 2. 
An analysis of simple main effects revealed that a significant choose-short bias occurred at the 5 -sec $[F(1,21)=$ $5.01, p<.05], 10-\sec [F(1,21)=7.71, p<.05]$, and $15-\sec [F(1,21)=5.07, p<.05]$ delay intervals, but not at the 0 -sec delay interval $[F(1,21)=2.62]$.

\section{Discussion}

The results of Experiment 2 support the hypothesis that the birds' differential outcome expectancies in Experiment 1 had detracted from the control exerted by the sample stimuli. On the first nondifferential test session, the DO group showed lower matching accuracy relative to the NDO group, as well as relative to its baseline performance. This finding suggests that the DO group had not been utilizing sample-stimulus information to the same extent as had the NDO group, apparently because its choice behavior had been more directly controlled by the expectancies of different outcomes. When the differential outcome expectancies were no longer available, the samples alone in the DO group were unable to support the same level of accuracy as they did in the NDO group. However, the samples rapidly reacquired control of choice behavior in the DO group, as evidenced by the equivalent accuracy in the DO and NDO groups on Sessions 2-10 of nondifferential testing. There was further evidence of the reemergence of control by sample stimuli in the subsequent delay tests, on which the DO group showed a significant choose-short effect equivalent to that shown by the NDO group.

Although the main thrust of the results is similar to that reported by Urcuioli (1990b), there are some differences in detail. First, the difference in accuracy between the DO and NDO groups during nondifferential test sessions dissipated much more rapidly in the present study than in Urcuioli's study. In addition, during delay tests following nondifferential test sessions, Urcuioli reported that the accuracy differential in favor of the NDO group reappeared. This was not observed in the present study. The delay test following nondifferential training demonstrated equivalent performance in the NDO and DO groups. Procedural differences in the two studies (P. J. Urcuioli, personal communication, April 20, 1992) may account for the variation in durability of the between-group effects. In the present study, pigeons had been trained to match on the basis of samples prior to the introduction of the redundant expectancy cue, whereas this did not occur in Urcuioli's (1990b) study. Consequently, the nondifferential test phase of the current study was the second time our birds were required to match on the basis of the samples alone, whereas it was the first time for the birds in Urcuioli's study.

\section{GENERAL DISCUSSION}

In Experiment 1, the performance of the DO groups was superior overall to that of the NDO group on both the temporal- and the nontemporal-sample dimension during delay testing. Although this replicates numerous other studies with respect to nontemporal samples, it provides more convincing evidence than do Santi et al. (1992) of a DOE effect on overall accuracy for temporal samples. Although the NDO group displayed a typical choose-short effect during delay testing in Experiment 1, the data for the DO groups indicated that they had a bias to select the comparison stimulus associated with the food outcome. The retention functions obtained for temporal samples with food and no-food outcomes in the present study were quite different from those obtained by Santi et al. (1992) with different probabilities of reward as the outcomes. In that study, there was no difference in zero-delay accuracy with temporal samples attributable to the different reinforcement-probability outcomes for either of the two DO groups, but there was for nontemporal samples. In addition, at delay intervals greater than $0 \mathrm{sec}$, the use of differential reinforcement probabilities resulted either in an increase in the choose-short effect (if the high probability was associated with short samples) or in an elimination of the choose-short effect (if the high probability was associated with the long sample). Thus, the retention functions for temporal samples differed markedly in the two DO groups and also differed from the retention functions for nontemporal samples.

In the present study, for both DO groups, there was a difference in accuracy between short and long samples at the 0-sec delay that could be attributed to a bias to select the comparison stimulus associated with the food outcome. This paralleled a similar bias seen with nontemporal samples at the 0-sec delay. In addition, at delays greater than $0 \mathrm{sec}$, both DO groups exhibited similar retention functions for temporal and nontemporal samples. These finding strongly suggest that when food and no food are used as outcomes for correct responding, temporal samples are not retrospectively and analogically coded. They appear to be coded primarily in terms of outcome expectancies. This interpretation was supported by the results of Experiment 2 . On the first nondifferential test session, the DO group showed a decrease in matching accuracy relative to both the NDO group and its previous baseline performance with differential outcomes. This finding suggests that when the differential outcome expectancies were no longer available, the samples alone in the DO group were unable to support the same level of matching accuracy as they did in the NDO group.

The main conclusion to be drawn from the present research is that temporal samples are not retrospectively and analogically coded when associated with food and no-food outcomes. Instead, both temporal and nontemporal sample stimuli are remembered in terms of their corresponding outcome expectancies. For nontemporal sample stimuli, these results replicate those reported by Urcuioli (1990b). For event durations as samples, these results support the notion of flexible coding of temporal information. Although analogical coding (Spetch \& Sinha, 1989; Wilkie \& Willson, 1990) may be the usual form of coding for event durations, a nonanalogical form of coding occurs when differential outcomes of food and no food are as- 
sociated with temporal samples. Santi and Mielke (1991) recently reported that when event durations are used as remember and forget cues for temporal samples, pigeons do not learn the reinforced discriminations by summing the duration of the sample and the duration of the temporal remember or forget cue. Rather, pigeons use the duration of the cue to determine whether the memory code for the temporal sample should be rehearsed. The present findings, along with those of Santi and Mielke (1991), provide further evidence of flexible coding of temporal information by pigeons.

\section{REFERENCES}

Brodigan, D. L., \& Peterson, G. B. (1976). Two-choice conditional discrimination performance of pigeons as a function of reward expectancy, prechoice delay, and domesticity. Animal Learning \& Behavior, 4, 121-124.

DeLong, R. E., \& Wasserman, E. A. (1981). Effects of differential reinforcement expectancies on successive matching-to-sample performance in pigeons. Journal of Experimental Psychology: Animal Behavior Processes, 7, 394-412.

Edwards, C. A., Jagielo, J. A., Zentall, T. R., \& Hogan, D. E. (1982). Acquired equivalence and distinctiveness in matching to sample by pigeons: Mediation by reinforcer-specific expectancies. Journal of Experimental Psychology: Animal Behavior Processes, 8, 244-259.

Honig, W. K., Matheson, W. R., Dodd, P. W. D. (1984). Outcome expectancies as mediators for discriminative responding. Canadian Joumal of Psychology, 38, 196-217.

Kraemer, P. J., Mazmanian, D. S., Roberts, W. A. (1985). The choose-short effect in pigeon memory for stimulus duration: Subjective shortening versus coding models. Animal Leaming \& Behavior, 13, 349-354.

Nevin, J. A., \& Grosch, J. (1990). Effects of signalled reinforcer magnitude on delayed matching-to-sample performance. Journal of Experimental Psychology: Animal Behavior Processes, 16, 298-305.

Peterson, G. B. (1984). How expectancies guide behavior. In H. L. Roitblat, T. G. Bever, \& H. S. Terrace (Eds.), Animal cognition (pp. 135-148). Hillsdale, NJ: Erlbaum.

Peterson, G. B., Trapold, M. A. (1980). Effects of altering outcome expectancies on pigeons' delayed conditional discrimination performance. Learning \& Motivation, 11, 267-288.

Peterson, G. B., Wheeler, R. L., \& Armstrong, C. D. (1978). Expectancies as mediators in the differential-reward conditional discrimination performance of pigeons. Animal Learning \& Behavior, 6, 279-285.

Peterson, G. B., Wheeler, R. L., \& Trapold, M. A. (1980). Enhancement of pigeons' conditional discrimination performance by expectancies of reinforcement and nonreinforcement. Animal Learning \& Behavior, 8, 22-30.
SANTI, A. (1989). Differential outcome expectancies and directed forgetting effects in pigeons. Animal Learning \& Behavior, 17, 349-354.

SANTI, A., Ducharme, M. J., Brudson, S. (1992). Differential outcome expectancies and memory for temporal and nontemporal stimuli in pigeons. Learning \& Motivation, 23, 156-169.

SANTI, A., \& MieLKe, M. (1991). Flexible coding of temporal information by pigeons: Event durations as remember and forget cues for temporal samples. Animal Leaming \& Behavior, 19, 171-176.

SANTI, A., Roberts, W. A. (1985a). Prospective representation: The effects of varied mapping of sample stimuli to comparison stimuli and differential trial outcomes on pigeons' working memory. Animal Leaming \& Behavior, 13, 103-108.

SANTI, A., RoBerts, W. A. (1985b). Reinforcement expectancy and trial spacing effects in delayed matching-to-sample by pigeons. Animal Learning \& Behavior, 13, 274-284.

SPETCH, M. L. (1987). Systematic errors in pigeons' memory for event duration: Interaction between training and test delay. Animal Learning \& Behavior, 15, 1-5.

SPETCH, M. L., Rusak, B. (1989). Pigeons' memory for event duration: Intertrial interval and delay effects. Animal Leaming \& Behavior, 17, 147-156.

SPETCH, M. L., \& Sinha, S. S. (1989). Proactive effects in pigeons' memory for event durations: Evidence for analogical retention. Joumal of Experimental Psychology: Animal Behavior Processes, 15, 347-357.

SPETCH, M. L., \& WILKIE, D. M. (1982). A systematic bias in pigeons' memory for food and light durations. Behaviour Analysis Letters, 2, 267-274.

SPETCH, M. L., \& Wilkie, D. M. (1983). Subjective shortening: A model of pigeons' memory for event duration. Joumal of Experimental Psychology: Animal Behavior Processes, 9, 14-30.

TRAPOLD, M. A. (1970). Are expectancies based upon different positive reinforcing events discriminably different? Learning \& Motivation, 1, 129-140.

URCulOU, P. J. (1990a). Differential outcomes and many-to-one matching: Effects of correlation with correct choice. Animal Learning \& Behavior, 18, 410-422.

URCuloli, P. J. (1990b). Some relationships between outcome expectancies and sample stimuli in pigeons' delayed matching. Animal Learning \& Behavior, 18, 302-314.

URCuIOLI, P. J. (1991). Retardation and facilitation of matching acquisition by differential outcomes. Animal Learning \& Behavior, 19, 29-36.

WILKIE, D. M., \& WILLSON, R. J. (1990). Discriminal distance analysis supports the hypothesis that pigeons retrospectively encode event duration. Animal Learning \& Behavior, 18, 124-132.

Williams, D. A., Butler, M. M., \& Overmier, J. B. (1990). Expectancies of reinforcer location and quality as cues for a conditional discrimination in pigeons. Journal of Experimental Psychology: Animal Behavior Processes, 16, 3-13.

(Manuscript received March 6, 1992; revision accepted for publication June 26, 1992.) 